\title{
Self-reported STIs and sexual health checks in a cross-sectional study of gay and bisexual men in New Zealand
}

\author{
Nigel Dickson, ${ }^{1}$ Adrian Ludlam, ${ }^{2}$ Peter Saxton, ${ }^{2}$ Anthony Hughes ${ }^{3}$
}

${ }^{1}$ Department of Preventive and Social Medicine, University of Otago, Dunedin, New Zealand ${ }^{2}$ Department of Social and Community Health, University of Auckland, Auckland, New Zealand

${ }^{3} \mathrm{New}$ Zealand AIDS

Foundation, Auckland, New Zealand

\section{Correspondence to}

Dr Nigel Dickson, Department of Preventive and Social

Medicine, University of Otago, PO Box 913, Dunedin 9054,

New Zealand;

nigel.dickson@otago.ac.nz

Received 5 December 2013 Revised 29 May 2014 Accepted 5 July 2014 Published Online First 23 July 2014
CrossMark

To cite: Dickson $\mathrm{N}$,

Ludlam A, Saxton $\mathrm{P}$, et al.

Sex Transm Infect

2015;91:49-54.

\section{ABSTRACT}

Objectives To determine the incidence of self-reported sexually transmitted infections (STIs) and sexual health checks in community and internet samples of New Zealand gay, bisexual and other men who have sex with men (MSM) and factors associated with these.

Methods We analysed anonymous self-completed data from 3138 MSM who participated in the location-based Gay Auckland Periodic Sex Survey (GAPSS) and the internet-based Gay Online Sex Survey (GOSS) undertaken in February 2011.

Results Overall $8.2 \%$ of the participants reported at least one STI in the previous 12 months, which did not differ significantly by demographic factors or HIV status. While having anal sex and more partners were associated with more STI, after adjustment for the number of partners, the type of partner (regular or casual) was not. Medium and low condom users reported STIs more than high condom users, regardless of partner type. Overall $40 \%$ had a sexual health checkup without an STI diagnosed in the past year, with similar numbers attending general practice and sexual health clinics. Having a check-up was lower among Pacific and Asian men, those identifying as bisexual and recruited online. While those with more partners, having anal intercourse and diagnosed with HIV were more likely to go for a check-up, those using condoms less often were not.

Conclusions STIs are commonly reported in this community sample of MSM but will underestimate the true incidence due to asymptomatic infection. Screening for STIs outside sexual health clinics should be normalised for MSM and made accessible, safe and relevant.

\section{INTRODUCTION}

In New Zealand, while gay, bisexual and other men who have sex with men (MSM) face an overwhelmingly higher burden of HIV than heterosexual men and women, ${ }^{1}$ less is known about how they are affected by other sexually transmitted infections (STIs). Routine STI surveillance is based on conditions seen at sexual health clinics (SHCs) and laboratory diagnosed Chlamydia trachomatis and Neisseria gonorrhoeae; however, neither of these sources report the numbers with same-sex behaviour. While recent centre-based studies and case reports have revealed elevated diagnoses of gonorrhoea, syphilis and Lymphogranuloma venereum infection among MSM in New Zealand, ${ }^{2}{ }^{3}$ there has been no such study in a large community-based sample. In addition, there is no information on how frequently sexually active MSM in New Zealand have sexual health checks despite recent guidelines recommending they have these annually. ${ }^{4}$

The broad approach to the control of infectious diseases in populations, including those passed on through sexual activity, is to minimise both their prevalence and the three general drivers of further spread: infectivity during contact, mixing between infected and uninfected and the duration of infection. ${ }^{5}$ For an individual, STI risk can be minimised through careful and consistent condom use and partner reduction, and for some immunisation. If still acquired, it might be possible to limit the impact by early detection and treatment. Not only does the lack of data on the current situation regarding sexual health checks make the development of appropriate recommendations in these areas difficult, but progress on control cannot be measured.

An improved focus on STIs among MSM has recently been urged. ${ }^{6}$ Internationally there are few community-based studies reporting STIs in these populations, which are most likely to be less common than in SHC-based populations. Information on self-reported STIs, sexual health checks and behaviour was collected in recent New Zealand community and internet-based behavioural surveillance among MSM, in which the prevalence of HIV was $6.5 \%$ in the community arm. ${ }^{7}$ The aim of this analysis is to examine the extent of selfreported STIs and screening behaviour in this large and diverse sample, and how these are related to sexual behaviour and demographic characteristics.

\section{METHOD}

We analysed data collected from the 2011 round of the Gay Auckland Periodic Sex Survey (GAPSS) and Gay Online Sex Survey (GOSS), an established behavioural surveillance system. ${ }^{8}$ GAPSS participants were recruited in Auckland, New Zealand, during 1 week in February 2011 from a gay community fair day and subsequently at all gay bars and sex-on-site venues. Eligibility criteria were being male, aged at least 16 years old, had sex with a man in the past 5 years and had not participated in GAPSS or GOSS that year. Questionnaires were anonymous and self-completed. Following GAPSS, the same questionnaire was used for the internetbased nationwide GOSS over the next month that accessed participants through banners on New Zealand internet dating sites. 
The two main outcomes of interest were responses to questions (a) "Have you been for a sexual health check-up or treatment in the last 12 months for any STIs", which was followed by options of 'no', or four possible commonly used sites they might have attended; and (b) "In the last 12 months have you had any of the following sexually transmitted infections?" that was followed by a list of the common STIs.

Participants were also asked the number, type (casual or regular) and nature of current regular relationships ("boyfriend/ long-term lover/life partner/civil union partner" or "fuckbuddy/ friend I have sex with"). Those who had engaged in anal intercourse were separately asked about condom use with their main regular and/or casual partners. Data on condom use were collected on a five-level scale that was collapsed into three: low ('very rarely' or 'never'), medium ('about half the time') and high ('always' or 'almost always'). Sociodemographic information asked included age, sexual identity, ethnicity, highest level of education and place of residence.

Statistical analyses were carried out using Stata V.12.1. Univariate analysis was conducted on demographic characteristics and behavioural data possibly associated with STI acquisition. ORs were calculated by fitting a logistic regression model of the dependent variable on the independent variable of interest. While ORs were adjusted for likely confounders, a model predicting STI risk was not created as the behavioural surveillance questionnaire was not designed to collect data on all potential predictors. ORs and adjusted ORs (AORs) are presented with $95 \%$ CIs. Non-responders were excluded from the relevant analyses.

The Ministry of Health's Northern $\times$ Regional Ethics Committee gave ethical approval.

\section{RESULTS}

Of the 3138 respondents, $257(8.2 \%)$ reported at least one STI in the previous 12 months. The most common was chlamydia (3.4\%), then gonorrhoea $(2.1 \%)$, genital or anal warts $(1.9 \%)$, genital or anal herpes (1.1\%), syphilis $(1.1 \%)$, with a small number reporting non-specific urethritis $(0.8 \%)$ and LGV $(0.1 \%)$. Reports did not vary significantly by age, residence, ethnicity or highest qualification, nor by whether the respondent was recruited online or offline $(8.1 \%$ vs $8.4 \%$, OR $0.96(0.74$ to 1.2$)$ ) or in the latter sample by site of recruitment (table 1 ).

A clear trend was found for increased STI reporting with more sexual partners (table 2); compared with those with one partner in the past 6 months those with $6-10$ had an AOR of 2.4 (1.5 to 4.0), and those with more than 50 an AOR of 5.4 (2.7 to 10.9). Men having anal sex were at higher risk, which remained after adjustment for number of sexual partners (AOR 2.3 (1.3 to 4.0$)$ ). Those having only regular partners had the same incidence of reported of STIs as those with only casual partners. Having both regular and casual partners doubled the unadjusted odds of an STI; however, once adjustment had been made for the total number for partners these men did not have an increased risk (AOR 0.93 (0.46 to 1.9)). Risk did not differ between men who were in a boyfriend-type relationship with their only or most frequent regular partner and those who said he was a friend they had sex with (AOR 0.90 (0.61 to 1.3)).

Among men having anal sex with regular partners, after adjustment for total number of partners, those with low condom use had double the odds (AOR 2.0 (1.3 to 2.0)) of an STI compared with those with high use. Similarly, among those with casual partners, there was an increased risk among those with medium condom use (AOR 1.8 (1.2 to 2.6)). When the categories of medium and low condom use were combined
Table 1 Proportion reporting an STI in the past 12 months by selected sociodemographic characteristics and site of enrolment in survey ( $\mathrm{N}=3138)$ (missing values for each variable not shown)

\begin{tabular}{|c|c|c|c|}
\hline & $\mathrm{N}$ & $\begin{array}{l}\text { Any STI } \\
\mathbf{n}(\%)\end{array}$ & Unadjusted OR \\
\hline \multicolumn{4}{|l|}{ Age (years) } \\
\hline $16-29$ & 1243 & $89(7.1)$ & Ref. \\
\hline $30-44$ & 1032 & $93(9.0)$ & $1.3(0.95$ to 1.7$)$ \\
\hline 45 or more & 836 & $75(9.0)$ & $1.3(0.93$ to 1.8$)$ \\
\hline \multicolumn{4}{|l|}{ Ethnicity } \\
\hline New Zealand European & 2262 & $193(8.5)$ & Ref. \\
\hline Maori & 317 & $29(9.1)$ & $1.1(0.72$ to 1.6$)$ \\
\hline Pacific & 83 & $4(4.8)$ & 0.5 (0.20 to 1.5$)$ \\
\hline Asian & 258 & $16(6.2)$ & 0.7 (0.42 to 1.2$)$ \\
\hline Other & 186 & $12(6.5)$ & 0.7 (0.40 to 1.4$)$ \\
\hline \multicolumn{4}{|l|}{ Sexual identity } \\
\hline Gay/homosexual & 2319 & $212(9.1)$ & Ref. \\
\hline Bisexual & 679 & $36(5.3)$ & $0.6(0.39$ to 0.80$)$ \\
\hline Other & 133 & $9(6.8)$ & 0.7 (0.36 to 1.4$)$ \\
\hline \multicolumn{4}{|l|}{ Highest qualification } \\
\hline No school qualification & 201 & $15(7.5)$ & 0.8 (0.48 to 1.5$)$ \\
\hline School only & 568 & $49(8.6)$ & $1.0(0.69$ to 1.4$)$ \\
\hline Professional or trades & 1067 & $78(7.3)$ & 0.8 (0.60 to 1.1$)$ \\
\hline University degree & 1260 & $111(8.8)$ & Ref. \\
\hline \multicolumn{4}{|l|}{ Place of residence } \\
\hline Auckland & 1654 & $138(8.3)$ & Ref. \\
\hline Elsewhere in New Zealand & 1304 & $103(7.7)$ & 0.9 (0.70 to 1.2$)$ \\
\hline Overseas & 105 & $42(13.3)$ & 1.7 (0.94 to 3.0$)$ \\
\hline \multicolumn{4}{|l|}{ Survey type and site } \\
\hline Offline-community event & 962 & $77(8.0)$ & Ref. \\
\hline Offline-bars & 118 & $11(9.3)$ & 1.2 (0.61 to 2.3 ) \\
\hline Offline-sex-on-site venues & 175 & $17(9.7)$ & $1.2(0.71$ to 2.1$)$ \\
\hline Online survey (GOSS) & 1883 & $152(8.1)$ & $1.0(0.76$ to 1.3$)$ \\
\hline
\end{tabular}

(table 2), there was a significant increase in STI risk in this group for both regular (AOR 1.8 (1.3 to 2.7)) and casual partners (AOR 1.7 (1.2 to 2.3)) compared with high condom users.

Men who had never tested for HIV were at significantly lower risk of reporting an STI than those who had last tested negative; this rose slightly after adjustment for number of sexual partners and anal sex (AOR $0.37(0.24$ to 0.56$)$ ). There was a non-significant increase in risk among those diagnosed with HIV compared with those who had last tested negative (AOR $1.3(0.74$ to 2.2$)$ ); that the OR dropped with adjustment for anal sex and number of partners indicated those with HIV infection had more such recent risk behaviours compared with those who had tested negative.

Overall 1544 (48.6\%) reported a sexual health check-up or STI treatment in the past 12 months. Of these, 1517 reported whether they had had an STI in the past year, and 237 (15.3\%) had. It is therefore assumed $1280(40.3 \%)$ had a sexual health check-up without an STI diagnosed in the past year, suggesting that the motivation for the attendance was to be checked. Of those, 741 (48.0\%) attended their general practitioner, 685 (44.4\%) an SHC, 137 (8.9\%) a New Zealand AIDS Foundation clinic and 74 (5.3\%) somewhere else; some men attending more than one site. Those who attended an SHC were more likely to report being diagnosed with an STI than those attending elsewhere.

The proportion having a check-up but no STI was lower among Pacific (AOR 0.61 (0.37 to 1.00)) and Asian men (AOR 
Table 2 Proportion reporting a sexually transmitted infection (STI) in past 12 months and association with selected behaviours in the past 6 months and known HIV status ( $\mathrm{N}=3138)$ (missing values for each variable not shown)

\begin{tabular}{|c|c|c|c|c|}
\hline & $\mathrm{N}$ & $\begin{array}{l}\text { Any STI } \\
\mathrm{n}(\%)\end{array}$ & Unadjusted OR (95\% Cl) & Adjusted OR (95\% Cl) \\
\hline Male partners & & & & * \\
\hline 0 & 230 & $10(4.3)$ & $0.94(0.45$ to 2.0$)$ & 1.7 (0.71 to 4.3$)$ \\
\hline 1 & 627 & $29(4.6)$ & Ref. & Ref. \\
\hline $2-5$ & 1177 & $76(6.5)$ & 1.4 (0.92 to 2.2$)$ & $1.2(0.77$ to 2.0$)$ \\
\hline $6-10$ & 456 & $54(11.8)$ & 2.8 (1.7 to 4.4$)$ & $2.4(1.5$ to 4.0$)$ \\
\hline $11-20$ & 293 & $29(9.9)$ & 2.3 (1.3 to 3.9$)$ & 2.0 (1.1 to 3.4$)$ \\
\hline $21-50$ & 195 & $36(18.5)$ & 4.7 (2.7 to 8.0$)$ & $4.0(2.3$ to 6.8$)$ \\
\hline$>50$ & 65 & $15(23.1)$ & 6.2 (3.1 to 12.5$)$ & $5.4(2.7$ to 10.9$)$ \\
\hline Male partners & & & & $t$ \\
\hline None & 240 & $10(4.2)$ & $0.76(0.36$ to 1.6$)$ & 0.85 (0.002 to 356$)$ \\
\hline Only regular & 445 & $24(5.4)$ & Ref. & Ref. \\
\hline Regular and casual & 1833 & $188(10.3)$ & 2.0 (1.3 to 3.1$)$ & $0.93(0.46$ to 1.9$)$ \\
\hline Only casual & 538 & $28(5.2)$ & $0.96(0.55$ to 1.7$)$ & $0.58(0.26$ to 1.3$)$ \\
\hline Any anal sex & & & & $\ddagger$ \\
\hline Yes & 2291 & $218(9.5)$ & 2.7 (1.7 to 4.2$)$ & $2.3(1.3$ to 4.0$)$ \\
\hline No & 639 & $24(3.8)$ & Ref. & Ref. \\
\hline Regular partners and condom use & & & & $\ddagger$ \\
\hline No anal sex & 186 & $8(4)$ & 0.51 (0.59 to 1.2$)$ & 0.98 (0.67 to 1.4$)$ \\
\hline High condom use & 554 & $45(8)$ & Ref. & Ref. \\
\hline Medium/low condom use & 696 & $88(13)$ & 1.6 (1.1 to 2.4$)$ & 1.8 (1.3 to 2.7$)$ \\
\hline No regular partner/NS & 1702 & 116 & & \\
\hline Casual partners and condom use & & & & $\ddagger$ \\
\hline No anal sex & 437 & $17(3.9)$ & 0.59 (0.41 to 0.86 ) & 0.54 (0.32 to 0.92$)$ \\
\hline High condom use & 1396 & $122(8.7)$ & Ref. & Ref. \\
\hline Medium/low condom use & 481 & $71(15)$ & 1.8 (1.3 to 2.5$)$ & $1.7(1.2$ to 2.3$)$ \\
\hline No casual partner/NS & 824 & 47 & & \\
\hline HIV status at last HIV test & & & & $t$ \\
\hline HIV positive & 130 & $19(15)$ & 1.6 (0.95 to 2.6$)$ & $1.3(0.74$ to 2.2$)$ \\
\hline HIV negative & 2096 & $205(10)$ & Ref. & Ref. \\
\hline Never tested/no result & 869 & $27(3)$ & 0.30 (0.20 to 0.45 ) & $0.37(0.24$ to 0.56$)$ \\
\hline
\end{tabular}

0.60 (0.44 to 0.81$)$ ), those identifying as bisexual (AOR 0.74 (0.61 to 0.91$)$ ), recruited online (AOR $0.71(0.59$ to 0.85$)$ ), with least formal education after also adjusting for age (AOR $0.63(0.44$ to 0.90$))$, and those who had never had an HIV test (AOR 0.12 (0.10 to 0.16$)$ ) (tables 3 and 4). Checks were commoner among those with more sexual partners, having anal intercourse (AOR 1.9 (1.5 to 2.4)) and those who had tested HIV positive (AOR 1.7 (1.1 to 2.5)) (table 4).

\section{DISCUSSION}

One in 12 gay and bisexual men in this New Zealand community-based and internet dating-site sample reported one or more STIs in the previous year. As expected this was more common among those having more sexual partners, and having anal intercourse, among whom regular condom use provided some protection. The number, rather than type of partner, was more important in predicting risk. The proportion with diagnosed HIV reporting an STI was not significantly higher than other MSM. Around $40 \%$ of the sample had a sexual health check without an STI being diagnosed in the past year, with similar numbers attending a general practitioner and SHC. Checks were more common among men having more partners, anal intercourse and those diagnosed with HIV, however was not with lower condom use; they were less common among Pacific and Asian men, those identifying as bisexual and enrolled online. While men with more sexual partners and having anal sex were more likely to have had a check in the previous year, this is unlikely to fully explain their higher incidence of reported STIs as the difference in STI diagnoses was greater than that of testing.

Limitations include reliance on self-reports, lack of information on the frequency of sexual contact and that information was sought on STIs in the past 12 months and behaviour over the previous six. The sample was obtained at a number of settings where gay and bisexual men congregate in Auckland, including the largest gay community event and popular internet dating sites. While it may not be representative of all MSM there or nationally, being obtained through a number of sites and approaches will make it more diverse. In the UK, MSM in a probability survey reported fewer STIs than those enrolled in both internet and community-based behavioural surveys. ${ }^{9}{ }^{10}$ Similarly, men in our sample with diagnosed HIV may not be typical of all infected. Our results will underestimate the true prevalence of infection among gay and bisexual men since many STIs are asymptomatic, ${ }^{11}$ some men with symptoms may not have sought or been offered care and tests may not have been comprehensive. 
Table 3 Variation in reporting a sexual health check among those who did not report a sexually transmitted infection (STI) in the previous 12 months by demographic characteristics $\left(\mathrm{N}=2868^{*}\right)$ (missing values for each variable not shown)

\begin{tabular}{|c|c|c|c|c|}
\hline & $\mathbf{N}$ & $\begin{array}{l}\text { Check-up <12 months } \\
\mathrm{n}(\%)\end{array}$ & Unadjusted OR $(95 \% \mathrm{CI})$ & Adjusted OR $(95 \% \mathrm{Cl})$ \\
\hline Age (years) & & & & $t$ \\
\hline $16-29$ & 1148 & $510(44)$ & Ref. & Ref. \\
\hline $30-44$ & 937 & $445(47)$ & $1.1(0.95$ to 1.3$)$ & $1.1(0.90$ to 1.3$)$ \\
\hline 45 or more & 756 & $316(42)$ & $0.90(0.75$ to 1.1$)$ & 0.85 (0.69 to 1.04$)$ \\
\hline Ethnicity & & & & $t$ \\
\hline New Zealand European & 2059 & $927(45)$ & Ref. & Ref. \\
\hline Maori & 287 & $143(50)$ & $1.2(0.95$ to 1.6$)$ & $1.2(0.92$ to 1.6$)$ \\
\hline Pacific & 77 & $28(36)$ & $0.70(0.43$ to 1.1$)$ & $0.61(0.37$ to 1.00$)$ \\
\hline Asian & 242 & $84(35)$ & 0.65 (0.49 to 0.86$)$ & $0.60(0.44$ to 0.81$)$ \\
\hline Other & 174 & $89(51)$ & $1.3(0.94$ to 1.7$)$ & $1.2(0.90$ to 1.7$)$ \\
\hline Sexual identity & & & & $\dagger$ \\
\hline Gay/homosexual & 2349 & $1210(52)$ & Ref. & Ref. \\
\hline Bisexual & 678 & $271(40)$ & 0.65 (0.54 to 0.79$)$ & $0.74(0.61$ to 0.91$)$ \\
\hline Other & 133 & $60(45)$ & $0.78(0.54$ to 1.1$)$ & $1.02(0.67$ to 1.6$)$ \\
\hline Highest qualification & & & & $\ddagger$ \\
\hline No school qualification & 185 & 68 (37) & $0.63(0.45$ to 0.86$)$ & $0.63(0.44$ to 0.90$)$ \\
\hline School only & 517 & $227(44)$ & 0.84 (0.68 to 1.04$)$ & $0.92(0.73$ to 1.2$)$ \\
\hline Professional or trades & 986 & $420(43)$ & $0.80(0.67$ to 0.95$)$ & 0.81 (0.67 to 0.97$)$ \\
\hline University degree & 1143 & $550(48)$ & Ref. & Ref. \\
\hline Place of residence & & & & $\dagger$ \\
\hline Auckland & 1828 & $911(50)$ & Ref. & Ref. \\
\hline Elsewhere in New Zealand & 1205 & $563(47)$ & 0.89 (0.76 to 1.03$)$ & $0.86(0.73$ to 1.01$)$ \\
\hline Overseas & 95 & $57(60)$ & $1.5(0.95$ to 2.3$)$ & 1.6 (1.0 to 2.6$)$ \\
\hline Survey type and site & & & & $\dagger$ \\
\hline Offline-community event & 884 & $414(47)$ & Ref. & Ref. \\
\hline Offline-bars & 107 & $56(52)$ & $1.2(0.83$ to 1.9$)$ & $1.0(0.64$ to 1.6$)$ \\
\hline Offline-sex-on-site venues & 158 & $79(50)$ & $1.1(0.81$ to 1.6$)$ & $0.86(0.59$ to 1.3$)$ \\
\hline Online survey (GOSS) & 1719 & $731(43)$ & $0.84(0.71$ to 0.99$)$ & 0.71 (0.59 to 0.85$)$ \\
\hline
\end{tabular}

The true response rate cannot be determined; while around half of the approaches at the offline sites declined, individuals may have been approached multiple times over the study period, making the acceptance rate lower than the participation rate. ${ }^{7}$

Although there is no comparable contemporary data from the general population, STIs in the past year were more common in our sample than annually among all men in a New Zealand birth cohort from sexual debut to age $32 .{ }^{12}$ The most recent national survey of sexual behaviour undertaken over 20 years ago found just over half (54\%) of the MSM reported ever having an STI, more than five times that of all men, ${ }^{13}$ and in a 1996 national self-selected survey of 1852 gay and bisexual men, $37 \%$ reported ever having an STI. ${ }^{12}$

International comparisons are also hard to make. In combined community-based surveys of MSM in three Australian cities in 2012, 12.0\% reported an STI in the previous year. ${ }^{14}$ In a 2010 study in Scotland, among MSM attending gay bars, $13.2 \%$ reported any STI in the past year, somewhat higher than the $9.3 \%$ in our comparable group. In a 2007 Norwegian internet survey of MSM, 8\% reported gonorrhoea, syphilis, chlamydia and/or HIV in the past year, ${ }^{15}$ and a similar German study $7.1 \%$ reported gonorrhoea, syphilis, chlamydia infection and/or HCV. ${ }^{16}$ While these results tentatively suggest STIs are less common among MSM in New Zealand than in Australia and some northern European countries, but at comparable levels in others, sampling and testing differences need to be considered.

There are however comparable data on STI testing. The 2010 European MSM Internet Survey (EMIS) collected data from 40 cities, with the median for STI testing in the past year being $40 \%$ (range 9-48\%). ${ }^{17}$ The proportion (44\%) in London in this survey was comparable to ours $(43 \%)$ in our online sample. Among those we enrolled from bars, 52\% reported a check or treatment, a similar number to those reporting an STI test in the previous year in the Scottish study (54\%). Overall these results suggest that STI testing in New Zealand is similar to the UK, and both higher and lower than some other European countries.

As has been consistently found, STIs were more likely among those with more sexual partners and anal intercourse. The initial significant association between having both regular and casual partners and STIs was lost after adjusting for total number of partners. The similar risk regardless of relationship type, which is associated with different relationship length and frequency of sexual contact, could reflect the high underlying prevalence of STIs within gay male communities, and the high infectivity of many of the STIs, meaning transmission might often occur after only a few exposures.

Condoms appeared to have a protective effect on STIs. Although our cross-sectional methodology means we cannot 
Table 4 Variation in reporting a sexual health check among those who did not report an sexually transmitted infection (STI) in the previous 12 months by sexual behaviour in previous 6 months and known HIV status ( $\left.N=2668^{*}\right)$ (missing values for each variable not shown)

\begin{tabular}{|c|c|c|c|c|}
\hline & $\mathrm{N}$ & $\begin{array}{l}\text { Check-up }<12 \text { months } \\
\mathrm{n}(\%)\end{array}$ & Unadjusted OR (95\% Cl) & Adjusted OR (95\% Cl) \\
\hline Male partners & & & & $\dagger$ \\
\hline 0 & 220 & $50(23)$ & 0.49 (0.34 to 0.71$)$ & $0.86(0.57$ to 1.3$)$ \\
\hline 1 & 595 & $222(37)$ & Ref. & Ref. \\
\hline $2-5$ & 1098 & $489(45)$ & 1.3 (1.1 to 1.7$)$ & $1.4(1.2$ to 1.8$)$ \\
\hline $6-10$ & 399 & $196(49)$ & 1.6 (1.3 to 2.1$)$ & $1.6(1.3$ to 2.2$)$ \\
\hline $11-20$ & 263 & $149(57)$ & 2.2 (1.6 to 3.0$)$ & $2.2(1.6$ to 2.9$)$ \\
\hline $21-50$ & 158 & $103(65)$ & 3.1 (2.2 to 4.6$)$ & 3.1 (2.1 to 4.6$)$ \\
\hline$>50$ & 49 & $32(65)$ & $3.2(1.7$ to 5.9$)$ & $3.0(1.6$ to 5.6$)$ \\
\hline Male partners & & & & $\ddagger$ \\
\hline None & 230 & $52(23)$ & 0.43 (0.30 to 0.62 ) & $0.46(0.32$ to 6.7$)$ \\
\hline Only regular & 419 & $170(41)$ & Ref. & Ref. \\
\hline Regular and casual & 1637 & $829(51)$ & 1.5 (1.2 to 1.9$)$ & 0.89 (0.63 to 1.2$)$ \\
\hline Only casual & 508 & $198(39)$ & $0.94(0.72$ to 1.2$)$ & $0.62(0.43$ to 0.90$)$ \\
\hline Any anal sex & & & & $\S$ \\
\hline Yes & 2064 & $1027(50)$ & $2.4(2.0$ to 3.0$)$ & $1.9(1.5$ to 2.4$)$ \\
\hline No & 612 & $177(29)$ & Ref. & Ref. \\
\hline Regular partners and condom use & & & & $\S$ \\
\hline No anal sex & 178 & $67(38)$ & 0.47 (0.33 to 0.67$)$ & 0.54 (0.38 to 0.77$)$ \\
\hline High condom use & 506 & $284(56)$ & Ref. & Ref. \\
\hline Medium/low condom use & 605 & $296(49)$ & 0.75 (0.59 to 0.95 ) & 0.81 (0.64 to 1.04$)$ \\
\hline No regular partner/NS & 1579 & $633(40)$ & & \\
\hline Casual partners and condom use & & & & $\S$ \\
\hline No anal sex & 417 & $149(36)$ & $0.52(0.41$ to 0.60$)$ & 0.52 (0.42 to 0.66$)$ \\
\hline High condom use & 1271 & $654(57)$ & Ref. & Ref. \\
\hline Medium/low condom use & 406 & $205(50)$ & $0.96(0.77$ to 1.2$)$ & 0.96 (0.77 to 1.2$)$ \\
\hline No casual partner/NS & 774 & $272(35)$ & & \\
\hline HIV status at last HIV test & & & & $\ddagger$ \\
\hline HIV positive & 111 & $78(70)$ & $1.8(1.2$ to 2.7$)$ & $1.7(1.1$ to 2.5$)$ \\
\hline HIV negative & 1885 & $1073(57)$ & Ref. & Ref. \\
\hline Never tested/no result & 835 & $113(14)$ & 0.12 (0.09 to 0.15$)$ & $0.12(0.10$ to 0.16$)$ \\
\hline
\end{tabular}

identify the sequence of condom use and STI acquisition, the consistency of our finding across both casual and regular partner contexts suggests condoms reduced risk.

About half of the sample had a check in the past year. This was more common among those at higher STI risk through more partners and anal intercourse, among those tested HIV positive, but not among those who used condoms less frequently. Although again no comparable data on the heterosexual population exist, it is likely these are commoner among MSM, which may indicate that gay men have greater awareness of sexual health issues. Nonetheless, it falls short of the recent recommendation for all MSM to have at least an annual check. ${ }^{18}$ In our sample, Pacific and Asian men were less likely to be checked, as were those who identified as bisexual and with less education, deserving further investigation. Our findings support the recommendation that there should be more frequent testing among those with more partners, that condom use should be encouraged for STI as well as HIV prevention and that patients who report not using condoms regularly should be prompted to have more checks.

Just under half $(48 \%)$ of the sexual health check-ups or STI treatments occurred in general practice, highlighting the importance of these settings for MSM and the need for general practitioners to be aware of appropriate testing, including screening for rectal STIs. A higher proportion of those attending an SHC reported an STI than those who had been to a general practitioner. This difference might have been due to those more likely to actually have an STI going to an SHC, or different diagnostic practices. Problematically, a New Zealand qualitative study found not all MSM disclose their sexuality and/or sexual practices to their doctors, ${ }^{19}$ and a large survey found that around two-thirds of gay and bisexual men said their healthcare providers always or usually presume they are heterosexual. ${ }^{20}$ Non-heterosexual practice needs to be routinely explored in all medical encounters related to sexual matters.

Consideration of how to increase testing should also include discussion on extending testing to less traditional locations. In New Zealand, rapid HIV and syphilis testing is currently being undertaken by some non-governmental organisations that have established links with sexual health services, at sex-on-site venues. With the development of home HIV testing kits, not currently available in New Zealand, the possibility of more selftesting may arise in the future, and how this can be best used to improve sexual health of MSM should then be considered. 
The HIV-infected men in the sample, most of whom had been diagnosed more than 12 months previously, did not have significantly higher reported incidence of STIs. In view of the international experience that HIV-infected MSM are disproportionately diagnosed with other STIs, ${ }^{21}$ and that our point estimate of the OR among this group was raised but not statistically significantly so, we are cautious about suggesting that HIV-infected men either are or are not at increased risk in New Zealand. We found a higher rate of sexual health screening among this group that could partially explain more STIs being diagnosed. Irrespective of whether they have a higher incidence, STIs have important implications for those with HIV, both syphilis and HSV-2 can adversely impact on their HIV control ${ }^{5}{ }^{22}$; also HIV and STIs have heightened infectivity in the presence of coinfection, which can facilitate HIV and STI outbreaks. ${ }^{23}{ }^{24} \mathrm{~A}$ particular concern is that as HIV control strategies internationally emphasise treatment as prevention and pre-exposure prophylaxis over condom use, more STIs will result. ${ }^{5}$ The potential for antiretroviral treatment to impact on HIV spread may be overly optimistic where the effect of other STIs to increase HIV transmission has not been factored into modelling. ${ }^{25}$

The clear message from this study is there needs to be access to sexual health checks and STI treatment for MSM in an environment in which they can safely discuss their sexuality and behaviour. Furthermore, the sexual health of MSM should not be viewed in isolation from the social context. ${ }^{26}$ Internationally there is evidence that some MSM may delay seeking services because they fear discrimination. ${ }^{27}$ Ensuring laws and policies promote the basic human rights of sexual minorities and legally recognise same-sex partnerships, as has now occurred in New Zealand, are important structural and policy changes. A broad health sector approach removing the inequalities in gay and bisexual men's sexual health that includes engagement with key community groups, improving the collection of data on the health of MSM, and expanding the provision of evidence-based prevention-as well as evaluating the effectiveness of interventions-remains a priority if sexual health is to be improved for gay and bisexual men.

\section{Key messages}

- Sexually transmitted infections (STIs) are commonly reported in this community sample of gay and bisexual men.

- The number, more than the type, of sexual partners was most associated with STIs.

- Condom use was associated with reporting fewer STIs.

- Screening for STIs should increase and general practitioner services be safe, comprehensive and accessible for gay and bisexual men.

\section{Handling editor Jackie A Cassell}

Acknowledgements We would like to thank the men who participated in the study and the site operators for allowing us access and Dr Massimo Giola for helpful comments.

Contributors ND, PS, and AH designed the study. PS project managed the data collection, AL analysed the data and ND wrote the first draft. All authors contributed to the manuscript and approved the final version.

Funding The New Zealand Ministry of Health funded the studies but had no role in their implementation or analysis.

Competing interests None.
Ethics approval The New Zealand Ministry of Health's Northern $\times$ Regional Ethics Committee.

Provenance and peer review Not commissioned; externally peer reviewed.

\section{REFERENCES}

1 McAllister SM, Dickson NP, Sharples K, et al. Unlinked anonymous HIV prevalence among New Zealand sexual health clinic attenders: 2005-2006. Int J STD AIDS 2008;19:752-7.

2 Azariah S, Perkins N. Prevalence of sexually transmitted infections in men who have sex with men presenting to Auckland Sexual Health Service. NZ Med J 2010;23:46-54.

3 Robertson A, Azariah S, Bromhead C, et al. Case report: lymphogranuloma venereum in New Zealand. Sex Health 2008;5:369-70.

4 Best Practice Advisory Centre. A "how-to guide" for a sexual health check-up. http://www.bpac.org.nz/BPJ/2013/April/contents.aspx (accessed 27 Nov 2013).

5 Giesecke J. Modern infectious disease epidemiology. London: Edward Arnold, 1994.

6 Nardone A, Semaille C. We need to improve screening for sexually transmitted infections in men who have sex with men in Europe. Sex Transm Infect 2013;89:539-40.

7 Saxton PJ, Dickson NP, Griffiths R, et al. Actual and undiagnosed HIV prevalence in a community sample of men who have sex with men in Auckland, New Zealand. BMC Public Health 2012;12:92.

8 Saxton PJ, Dickson NP, Hughes AJ. Who is omitted from repeated offline HIV behavioural surveillance among MSM?: implications for interpreting trends. AIDS Behav 2013;17:3133-44.

9 Evans AR, Wiggins RD, Mercer $\mathrm{CH}$, et al. Men who have sex with men in Great Britain: comparison of a self-selected internet sample with a national probability sample. Sex Transm Infect 2007:83:200-5.

10 Dodds JP, Mercer CH, Mercey DE, et al. Men who have sex with men: a comparison of a probability sample survey and a community based study. Sex Transm Infect 2006;82:86-7.

11 Machalek DA, Poynten M, Jin F, et al. Anal human papillomavirus infection and associated neoplastic lesions in men who have sex with men: a systematic review and meta-analysis. Lancet Oncol 2012;13:487-500.

12 Paul C, van Roode T, Herbison P, et al. Longitudinal study of sexually transmitted infection acquisition by gender and age up to age 32. Sex Transm Dis 2009;36:63-9.

13 Saxton PJ, Hughes AJ, Robinson EM. Sexually transmitted diseases and hepatitis in a national sample of men who have sex with men in New Zealand. N Z Med J 2002;115:U106.

14 Holt M, Hull P, Lea T, et al. Comprehensive testing for, and diagnosis of, sexually transmissible infections among Australian gay and bisexual men: findings from repeated, cross-sectional behavioural surveillance, 2003-2012. Sex Transm Infect 2014;90:208-15.

15 Jakopanec I, Schimmer B, Grjibovski AM, et al. Self-reported sexually transmitted infections and their correlates among men who have sex with men in Norway: an Internet-based cross-sectional survey. BMC Infect Dis 2010;10:261.

16 Schmidt AJ, Marcus U. Self-reported history of sexually transmissible infections (STIs) and STI-related utilization of the German health care system by men who have sex with men: data from a large convenience sample. BMC Infect Dis 2011;11:132.

17 Schmidt AJ, Hickson F, Weatherburn P, et al. Comparison of the performance of STI Screening Services for gay and bisexual men across 40 European cities: results from the European MSM Internet Survey. Sex Transm Infect 2013;89:575-82.

18 Centers for Disease Control. Sexually Transmitted Disease Treatment Guidelines, 2010. http://www.cdc.gov/std/treatment/2010/specialpops.htm (accessed 27 Nov 2013).

19 Adams J, McCreanor T, Braun V. Doctoring New Zealand's gay men. N Z Med J 2008; 121:11-20.

20 Neville $S$, Henrickson M. Perceptions of lesbian, gay and bisexual people of primary healthcare services. J Adv Nurs 2006;55:407-15.

21 Dougan S, Evans BG, Elford J. Sexually transmitted infections in Western Europe among HIV-positive men who have sex with men. Sex Transm Dis 2007;34:783-90.

22 Duffus WA, Mermin J, Bunnell R, et al. Chronic herpes simplex virus type-2 infection and HIV viral load. Int J STD AIDS 2005;16:733e5.

23 Cohen MS, Hoffman IF, Royce RA, et al. Reduction of concentration of HIV-1 in semen after treatment of urethritis: Implications for prevention of sexual transmission of HIV-1. Lancet 1997;349:1868-73.

24 Cohen MS. When people with HIV get syphilis: Triple jeopardy. Sex Transm Dis 2006;33:149-50.

25 Kalichman SC, Pellowski J, Turner C. Prevalence of sexually transmitted co-infections in people living with HIVIAIDS: systematic review with implications for using HIV treatments for prevention. Sex Transm Infect 2011;87:183-90.

26 Wolitski RJ, Fenton KA. Sexual health, HIV, and sexually transmitted infections among gay, bisexual, and other men who have sex with men in the United States. AIDS Behav 2011;15:9-17.

27 Mahajan AP, Sayles JN, Patel VA, et al. Stigma in the HIV/AIDS epidemic: a review of the literature and recommendations for the way forward. AIDS 2008;22(Suppl 2): S67-79. 